submitted to ApJS

\title{
The Twenty-Five Year Lick Planet Search ${ }^{1}$
}

\author{
Debra A. Fischer ${ }^{2}$, Geoffrey W. Marcy ${ }^{3,4}$, Julien F. P. Spronck ${ }^{2}$ \\ debra.fischer@yale.edu
}

\begin{abstract}
The Lick planet search program began in 1987 when the first spectrum of $\tau$ Ceti was taken with an iodine cell and the Hamilton Spectrograph. Upgrades to the instrument improved the Doppler precision from about $10 \mathrm{~m} \mathrm{~s}^{-1}$ in 1992 to about $3 \mathrm{~m} \mathrm{~s}^{-1}$ in 1995 . The project detected dozens of exoplanets with orbital periods ranging from a few days to several years. The Lick survey identified the first planet in an eccentric orbit (70 Virginis) and the first multi-planet system around a normal main sequence star (Upsilon Andromedae). These discoveries advanced our understanding of planet formation and orbital migration. Data from this project helped to quantify a correlation between host star metallicity and the occurrence rate of gas giant planets. The program also served as a test bed for innovation with testing of a tip-tilt system at the coudé focus and fiber scrambler designs to stabilize illumination of the spectrometer optics. The Lick planet search with the Hamilton spectrograph effectively ended when a heater malfunction compromised the integrity of the iodine cell. Here, we present more than 14,000 velocities for 386 stars that were surveyed between 1987 and 2011.
\end{abstract}

Subject headings: surveys, techniques: radial velocities

\footnotetext{
${ }^{1}$ Based on observations obtained at the Lick Observatory, which is operated by the University of California

${ }^{2}$ Department of Astronomy, Yale University, New Haven, CT USA 06520

${ }^{3}$ Department of Astronomy, University of California, Berkeley, CA USA 94720-3411

${ }^{4}$ Department of Physics and Astronomy, San Francisco State University, San Francisco, CA, USA 94132

${ }^{5}$ UCO/Lick Observatory, University of California at Santa Cruz, Santa Cruz, CA, USA 95064
} 


\section{Introduction}

Humans have long been curious about the existence of planets orbiting other stars and steady technological advances over the past half century resulted in remarkable progress that enabled the detection of hundreds of exoplanets. Struve (1952) was first to suggest that even then state of the art radial velocity measurements might be used to detect putative gas giant planets if they orbit close to their host stars. Twenty years later, Griffin \& Griffin (1973) outlined a strategy for achieving high Doppler precisions; they emphasized that the key was to superimpose a wavelength standard in the stellar spectrum so that instrumental shifts would equally affect both the calibrator and the stellar observations. They pointed out that telluric lines could serve in this role and estimated that Doppler shifts of $10 \mathrm{~m} \mathrm{~s}^{-1}$ could be measured with the limiting precision set by pressure, temperature and wind variations in the Earths atmosphere.

Campbell \& Walker (1979) designed an ingenious version of telluric lines in a bottle, filling a $0.90-\mathrm{m}$ long glass cell with hydrogen fluoride (HF) gas and positioning the cell in front of the slit of a coudé spectrograph at the 3.6-m Canada France Hawai'i telescope (CFHT). This pioneering technique was ideal for a general purpose spectrograph because the HF absorption lines were superposed on the stellar spectrum. By virtue of tracing out an identical light path, the HF wavelength calibration was imprinted with the same instrumental profile as the stellar spectrum. The team launched a survey of 16 stars during 27 observing runs (of three or fewer nights each) spanning six years. They used a spectrograph flux meter to derive accurate photon-weighted midpoints for their exposure times and calculated runto-run corrections of tens of meters per second for zero-point variations (Campbell, Walker \& Yang 1988). The team also modeled the effect of asymmetry variations in the instrumental profile of the spectrograph (Campbell \& Walker 1985). In this sample of 16 stars, Campbell, Walker \& Yang (1988) identified residual velocity variations to the binary orbit of $\gamma$ Cephei (Walker et al. 1992). Although this velocity scatter was initially attributed to stellar noise sources, Hatzes et al. (2003) later determined that these were reflex velocities induced by a gas giant planet. Fourteen stars showed internal errors of about $10 \mathrm{~m} \mathrm{~s}^{-1}$ and rms scatter of about $13 \mathrm{~m} \mathrm{~s}^{-1}$. Campbell, Walker \& Yang (1988) calculated upper limits to companion masses in the mass-period plane for the stars in their sample. While six stars (including $\epsilon$ Eridani) exhibited velocity scatter greater than $2.5 \sigma$, no exoplanets were detected in this small sample.

A significant body of work at the time (Dravins, Lindegren \& Nordlund 1981; Dravins 1982, 1985; Gray \& Toner 1985) indicated that stellar signals, including spots, p-mode oscillations and magnetically modulated granulation changes could introduce line profile variations that might be misinterpreted as Doppler shifts. The spectral format used by 
Campbell, Walker \& Yang (1988) did not include the Ca II H\&K lines, known to be a good diagnostic of chromospheric activity. Instead, they monitored the equivalent width of the 8662 A Ca II infrared triplet line for line filling from chromospheric emission. Coherent variability was measured in the equivalent widths of the $8662 \AA$ line for some of their target stars, however, no correlations were found between this activity metric and the measured radial velocities. Campbell, Walker \& Yang (1988) also implemented an observing strategy to deal with p-mode oscillations identified by Noyes et al. (1984) in $\epsilon$ Eridani: to average over these oscillations, the exposure time for this star was set to be a multiple of the 10-minute oscillation period.

There were serious challenges with the HF reference cell. The HF lines were pressure sensitive and the cell had to be run at the high temperature of $100^{\circ} \mathrm{C}$. A relatively long 0.9-m path length was required to provide a sufficient column density of HF molecules and the cell had to be refilled for each observing run. Perhaps most important, HF is a dangerous toxic substance. Other reference cells had also been developed and showed equal promise in delivering high precision. Iodine cells were a particularly promising choice and provided a wavelength standard that had long been used in lock-in amplifiers to stabilize lasers. Beckers (1977) used an iodine reference cell in the light path of a spectrometer to measure the relative Doppler shift for a single Ti II line (5713.9 $\AA$ ) in the solar umbra; this technique yielded a precision of $15 \mathrm{~m} \mathrm{~s}^{-1}$. Libbrecht $(1988,1989)$ later used an iodine reference cell to measure stellar p-mode oscillations at the Palomar coudé spectrograph.

At about the same time, McMillan et al. (1986) investigated the use of a Fabry-Pérot interferometer to measure stellar Doppler shifts induced by orbiting planets. They used optical fibers to couple starlight into an echelle spectrograph to minimize the effects of guiding and seeing on the instrumental profile and injected a comb of etalon lines with a finesse of 12, calibrated to argon and iron emission lines and tuned to compensate for the barycentric velocity at the telescope. Although the program did not detect any exoplanets, some of the innovations, in particular the scrambling of light with fibers and etalons for wavelength calibration, are being revisited with success today.

Cochran \& Hatzes (1990) constructed a prototype $\mathrm{I}_{2}$ cell and reported that their day sky tests in November and December 1988 at the 2.7-m McDonald telescope indicated that the iodine technique might be able to achieve routine Doppler measurements with precisions better than $5 \mathrm{~m} \mathrm{~s}^{-1}$ in stars with sufficient photospheric absorption lines. In a follow-up pilot program on the lower resolution 2.1-m coudé spectrograph at the McDonald Observatory, they used an iodine reference cell to measure the radial velocity shifts of a set of evolved giant stars. The spectral range for these measurements was only $23 \AA$, centered at 5530 $\AA$ and the iodine cell was not temperature controlled; at times, iodine was observed to 
condense out of its gaseous state. Although Cochran \& Hatzes (1990) commented on the importance of modeling the instrumental profile, they did not carry out this step. Despite these compromises in the early program, the pilot project achieved a velocity precision of 10 $-15 \mathrm{~m} \mathrm{~s}^{-1}$.

When Marcy \& Butler (1992) began a Doppler planet search at Lick Observatory in 1987, they elected to use an approach similar in principal to that of Campbell \& Walker (1979). They began tests of precise Doppler measurements with an iodine reference cell at the entrance slit of the Hamilton spectrograph (Vogt 1987) at Lick Observatory. Particular attention was paid to minimizing changes in the illumination of the spectrograph: a collimator mask was used to ensure that small movements of the telescope pupil with hour angle would not cause shifts in the position of the collimated beam on the downstream optics, the thorium argon lamp was focused each night with an algorithm to ensured that the same emission lines hit the same pixels on the CCD detector each night, and the alignment of the iodine cell with the telescope optical axis was checked at the beginning of each run. Perhaps the most important breakthrough was the successful modeling of the instrumental profile (Marcy \& Butler 1992; Valenti et al. 1995; Butler et al. 1996) also called the point spread function (PSF) or most accurately the spectral line spread function SLSF (Spronck et al. 2013). Marcy \& Butler (1992) developed a rigorous Doppler analysis code to model the observed spectrum; free parameters included shifts in the stellar lines from barycentric or orbital motion, the superimposed iodine lines, and the SLSF from the Hamilton spectrograph.

The $3 \mathrm{~m} \mathrm{~s}^{-1}$ breakthrough Doppler precision of the Lick planet search program allowed for the confirmation of radial velocity signals consistent with a gas giant planet orbiting

51 Peg b (Mayor \& Queloz 1995; Marcy et al. 1997) and for the detection of dozens of other exoplanets. In the early days, little time and very modest funding was allocated to the project, yet the Lick Planet Search ran for almost twenty five years, from 1987 until 2011. For all practical purposes, the project ended when the insulation around the iodine cell overheated and damaged the cell. Here, we present the radial velocity measurements that were obtained with that program.

\section{The Lick Planet Search}

The Hamilton spectrograph is a high-resolution optical echelle spectrometer that is located at the coudé focus of the 3-m Shane telescope with a second light feed from the Coudé Auxiliary Telescope (CAT), which is a siderostat-fed 0.6-m telescope. The CAT enables use of the spectrometer when other instruments are being used on the 3-m telescope. 
The free spectral range can include $3400 \AA$ to $9000 \AA$ in a single observation. The instrument resolution varies from 50,000 to 115,000 depending primarily on the choice of decker (width of the entrance slit).

\subsection{Doppler Analysis}

The Lick planet search began in 1987 June when an iodine cell was installed in front of

the entrance slit to the the coudé Hamilton spectrograph (Vogt 1987). The version of the Doppler code used to derive velocities for the stars presented here was developed by Paul Butler and Geoff Marcy and is described in detail in a series of papers: Marcy \& Butler (1992); Butler et al. (1996); Valenti et al. (1995). However, the Doppler analysis for more than 14,000 velocity measurements presented in this paper was rerun with a single version of the Doppler code, on a single computer and one version of IDL to ensure version control of subroutines, control of model inputs like the SLSF description and the templates and general consistency in the analysis before archiving the data.

The iodine absorption cell acts as a transmission filter, imprinting thousands of narrow iodine absorption lines on the stellar spectrum in the wavelength region from 5010 to $6200 \AA$. The iodine lines provide a fiducial wavelength scale and are used to model the wavelengthdependent SLSF of the echelle spectrometer. Because the SLSF changes over the echelle format of the CCD detector, the spectrum is broken into $2 \AA$ (40-pixel) chunks from 5010 to $5800 \AA$ (orders with telluric contamination were excluded in this version of the Doppler code, but are more surgically masked out in later versions).

The Doppler analysis is carried out for each of these chunks independently with a forward modeling technique. The Doppler model requires the true intrinsic stellar spectrum (ISS) for each of the target stars. The precursor to the ISS is a "template" spectrum of the program star obtained with high resolution and high signal-to-noise ratio (SNR) without the iodine cell. Because all spectra are broadened (often by an asymmetric SLSF), the template spectrum is deconvolved using a SLSF that is itself a model (see Section 3.2) that maps the high-resolution FTS spectrum of the cell to an echelle spectrum of the iodine cell (illuminated by a featureless B star). The B-star iodine cell observations bracket the template spectrum in time to minimize changes in the SLSF. One ISS is generated for every program star on the Lick Planet Search project. The ISS is then multiplied by an appropriate segment of the high-resolution FTS spectrum of the iodine cell and this product is convolved with a new SLSF model to fit the time-series spectra obtained with the iodine cell. The modeling process is driven by a Levenberg-Marquardt algorithm and the free parameters for each 40-pixel chunk include the wavelength zero point, dispersion, continuum normalization, a 
Doppler shift and eleven free parameters that describe the SLSF. Each chunk has its own SLSF model, wavelength solution and Doppler shift and differential velocities are calculated independently for each chunk over time. The nightly radial velocity is calculated as the weighted mean of the Doppler shifts for all of the chunks and the uncertainty is the standard deviation of the chunk velocities.

\section{Stability Challenges}

Over the twenty-five year time span of the Lick planet search there were many changes that were made to support the planet search project and the general user community. The instrumental profile was very asymmetric and the instrument resolution was $\mathrm{R} \sim 40,000$ until the optics of the Schmidt camera of the spectrometer were upgraded by installing a new corrector plate and field flattener in 1994 November. Before the camera upgrade, the single measurement Doppler precision for the Lick Planet Search was $\sim 10 \mathrm{~m} \mathrm{~s}^{-1}$. After the optics upgrade and an upgrade to a larger CCD detector, the Doppler precision improved to about $3 \mathrm{~m} \mathrm{~s}^{-1}$.

Before internet time was available, the current date and time were manually set each night on the Disk Operating System (DOS) computers by phoning to get a recorded message of the time. Before auto guiders were implemented, manual guiding of the star on the slit was done with an RA-Dec paddle (while viewing crosshairs through a periscope). The original slit and decker consisted of blades in front of a V-shaped jaw so that small variations in the

actual slit width could occur for mechanical reasons like backlash in the slit motor. The slit and decker system was replaced with an aperture plate in 1998 that presented a set of machined rectangular slits at the entrance to the spectrometer.

The Hamilton spectrograph is a facility instrument and the position of the spectral format on the detector can be adjusted with a motorized grating tilt or a change in the vertical position of the dewar. The instrument is regularly reconfigured by observers with different programs, introducing a significant source of instability for the precision Doppler program. To initialize the spectrometer setup, a focus routine is run at the beginning of each planet search night to insure that emission lines from the thorium argon lamp fall on the same pixel, giving a wavelength solution that is constant to roughly \pm 0.5 pixel.

Although the instrument is in a closed room at the coudé focus, the south-facing wall of the coudé room is warmed by direct sunlight. A weather station that monitored temperatures in the coudé room in 2001 recorded typical diurnal swings on the Celsius scale of a few degrees and seasonal changes of about ten degrees. The Hamilton spectrograph optics were mounted 
on an I-beam in the coudé room and would have experienced some thermal expansion and contraction. There was also significant variability in pressure and humidity. A dehumidifier operated during the summer months. In the winter, a stream of $\mathrm{N}_{2}$ gas flowed across the dewar window to mitigate condensation.

Precise corrections for the barycentric motion of the Earth are critical for accurate Doppler measurements. An error of 30 seconds in the exposure midpoint time contributes up to $1 \mathrm{~m} \mathrm{~s}^{-1}$ error in the measured stellar velocity. The geometrical midpoint of an exposure is only the right value to use if the conditions are perfectly clear. If the seeing is variable or if there is variable cloud coverage, then a photon-weighted midpoint should be used for calculating the barycentric velocity. To calculate the flux-weighted midpoint time, a photon integrator was used that predated the Hamilton spectrograph. The photon integrator consists of a propeller blade behind the slit that rotates at a few $\mathrm{Hz}$ and diverts about $5 \%$ of the light to a photomultiplier tube (Kibrick et al. 2006). A running photon count is displayed in the software interface so that the exposures could be terminated when the desired SNR was reached; this allows for better uniformity in the quality of spectra. An upgrade to the exposure meter allows for automatic termination of observations when a specified SNR is acquired. Even with an exposure-meter, barycentric errors can accumulate for subtle reasons, including small non-linear terms in the barycentric motion or sampling errors in the photon counter. To further limit errors in the photon-weighted midpoint time, exposure times are limited to a maximum of 20 minutes. If higher SNR is desired, velocities are determined for each observation and consecutive observations are averaged.

\subsection{CCD changes}

One of the most impactful changes to the Lick planet search project involved a series of upgrades of CCD detectors. The pixel size and charge transfer characteristics of the detector affect the spectral resolution and contribute to the SLSF so that CCD replacements can introduce velocity offsets that required zero-point corrections based on "standard" stars on the program (stars with low rms velocities). The initial commissioning of the Lick planet search in 1987 used a TI $800 \times 800$ pixel CCD. This detector was upgraded in 1990 to a $2048 \times 2048$ Ford Aerospace CCD detector, and the spectral format spanned $4850-6700 \AA$. Only the wavelength segments containing iodine (5090 - $5787 \AA)$ were used in the Doppler analysis, including slightly more than 300 chunks that were 40 pixels wide. None of these pre-upgrade observations were reanalyzed for this paper; they have been archived since 1997 with a zero-point velocity locked to data obtained with the replacement CCD for this project (Dewar 13, discussed below). 
The star $\tau$ Ceti was observed to have low rms scatter of $9.4 \mathrm{~m} \mathrm{~s}^{-1}$ by Campbell, Walker \& Yang (1988) and was regularly used as one of the radial velocities standards for the Lick planet search program. A total of 153 observations of $\tau$ Ceti were made with these first two detectors at Lick. The observations spanned 7 years from September 8, 1987 to Sept 27, 1994. The median error bar was $9.6 \mathrm{~m} \mathrm{~s}^{-1}$ with an unbinned velocity RMS of $12.3 \mathrm{~m} \mathrm{~s}^{-1}$. During this so-called pre-upgrade era at Lick (i.e., before the Schmidt camera optics were refurbished) nightly velocity corrections were made at the level of $10 \mathrm{~m} \mathrm{~s}^{-1}$ to account for zero-point errors from uncontrolled changes in the instrumental setup.

\subsection{Dewar 13}

In 1994 March (just before the Schmidt camera was upgraded in 1994 November), the Hamilton Spectrograph detector was upgraded and mounted in Dewar 13. By custom, the CCD is assigned the name of the dewar in which it is mounted. The detector specifications for this and all subsequent detectors are archived on the Mount Hamilton website 1 . The CCD in Dewar 13 was a $2048 \times 2048$ "Lick3" device with 15 -micron pixels and a quantum efficiency (QE) of about $23 \%$ over the iodine region. The full spectral format spanned from 4850 - $9362 \AA$ and there were 704 chunks in the iodine region, spanning 5090 to $5787 \AA$. Using Dewar 13, 94 observations of $\tau$ Ceti were obtained in the 3 years from 1994 November 18 to 1997 December 12 . The median error dropped from $9.6 \mathrm{~m} \mathrm{~s}^{-1}$ in the pre-upgrade era to $2.4 \mathrm{~m} \mathrm{~s}^{-1}$ with an RMS of $5.84 \mathrm{~m} \mathrm{~s}^{-1}$. The complete time series velocities for $\tau$ Ceti are plotted in Figure 1.

Two years of SLSF models for a particular central chunk in the iodine region of Dewar 13 observations are shown in Figure 2. These SLSF models were derived for spectra of featureless B stars observed from 1995 to 1997 with Dewar 13 at Lick Observatory. Because the B stars are inherently featureless and follow the same light path as our program stars, they simply serve to illuminate the iodine cell. Thus, the models in Figure 2 show the smearing function (SLSF) that transforms a high resolution FTS spectrum of iodine (Figure 3a) into our observed spectrum (Figure 3b). The taller narrower set of SLSF models in Figure 2 are fitted to spectra observed with the CAT 0.6-m telescope and the shorter and broader family of SLSF models are for spectra obtained with the 3-m Shane telescope. The Shane telescope and the CAT both have $\mathrm{f} / 36$ focal ratios, however the plate scale is 1.89 arcseconds per millimeter for the Shane and 9.45 arcseconds per millimeter for the CAT. This difference in the image magnification at the slit results in the different spectral line

\footnotetext{
${ }^{1}$ http://mthamilton.ucolick.org/techdocs/detectors
} 
spread function models in the observed spectra.

In addition to the two families of SLSF models in Figure 2 (CAT observations vs those from the 3-m Shane telescope), it is clear that there is substantial variation in the SLSF. Variability in the SLSF can be caused by changes in temperature and pressure in the spectrograph, guiding errors, differences in the motor-driven slit width or variability in seeing. The variability that is so apparent in the SLSF demonstrates that the Hamilton optics are not being illuminated in the same way. If the SLSF model is not accurate this is a source of instability that contributes to systematic errors in the Doppler measurements. Errors in the SLSF model can be introduced by cross-talk with the wavelength solution or by attempts to minimize $\chi^{2}$ by fitting noise.

A key advantage of the Dewar 13 CCD was the relatively narrow SLSF. The median resolution measured from thorium argon observations with the $1^{\prime \prime} .2$ slit was $R=64,000$. However, a significant disadvantage to the Dewar 13 detector was the low quantum efficiency.

\subsection{Dewar 6}

In February 1998, we upgraded to a "Lick-3" 2048× 2048 CCD that had been thinned by Michael Lesser to yield a QE of about $88 \%$ in the iodine region. This CCD was also assigned the name of the dewar in which it was mounted, Dewar 6. Unfortunately, the Dewar 6 device had a slightly concave shape and suffered from charge diffusion so that the SLSF was both more asymmetric and broader than Dewar 13. Four years of SLSF models fitted to B star illuminated iodine cell observations are shown for a particular wavelength chunk in Figure 4. The broader SLSF resulted in a drop in resolution to $\mathrm{R}=47,000$ when observing with the $1^{\prime \prime} .2$ slit.

We obtained 869 observations of the radial velocity constant star, $\tau$ Ceti, with the Dewar

6 detector. Most of the spectra were obtained over a four year period from July 30, 1998 to August 29, 2002. This detector was used once as an engineering back-up for an observing run in Jan 2006. The Doppler analysis was carried out for 704 40-pixel chunks spanning the wavelength range 5090 to $5787 \AA$. The median internal single measurement error was $2.93 \mathrm{~m} \mathrm{~s}^{-1}$ with a RMS of $6.31 \mathrm{~m} \mathrm{~s}^{-1}$. Thus, this particular detector was more efficient, but yielded a slightly poorer Doppler precision. 


\subsection{Dewar 8}

We were reluctant to make another upgrade to the detector because every major change to the instrument has a high probability of introducing systematic radial velocity offsets that compromise our ability to detect long period planets. However, in 2001, we were given the opportunity to try (without cost) a deep depletion device manufactured by LBNL. The silicon substrate in this chip was 200 microns thick, so a large voltage across the CCD was required to control lateral motion of the electrons (charge diffusion). Upon testing we found that we largely recovered the narrow and nearly symmetric SLSF of the Dewar 13 CCD, yielding a resolution of 56,000 while providing a QE of $75 \%$ in the iodine region. A representative set of SLSFs for the B star illuminated iodine cell observations taken from 2001 to 2011 (again for the same wavelength chunk) are plotted in Figure 5. We adopted this new CCD which was mounted into Dewar 8 at Lick Observatory. At the time that this detector change was made, we also increased the spectral format to include the $\mathrm{Ca}$ II H\&K lines as a chromospheric activity diagnostic.

\subsection{Dewar zero-point offsets}

Each of the program stars had a unique deconvolved template spectrum, or ISS (see Section 2.1), and that same ISS was used to model relative Doppler shifts for the star over the entire time span from 1995 to 2011. However, each CCD change can be accompanied by zero-point offsets in the derived velocities, in part because of changes to the SLSF. To check for zero-point offsets that could be introduced by the use of different detectors, we selected all stars with more than 100 observations taken between 1995 and 2011 and a velocity RMS less than $20 \mathrm{~m} \mathrm{~s}^{-1}$. We further required that at least 10 Doppler measurements were available with each of the three detectors (Dewar 13, Dewar 8, Dewar 6). Eighteen stars met these selection criteria; the typical number of observations was 30 for Dewar 13 and Dewar 6 and 90 observations for Dewar 8. We calculated the median velocity measured in each detector era for these "standard" stars to see if there were significant zero-point offsets. We found that the set of Dewar 13 velocities were on average only $0.37 \mathrm{~m} \mathrm{~s}^{-1}$ higher than the Dewar

6 velocities. However the mean Dewar 8 velocities were on average $13.1 \mathrm{~m} \mathrm{~s}^{-1}$ less than the Dewar 6 velocities.

A reality check comes from the extensive set of measurements for $\tau$ Ceti, which was one of the stars in the set of 18 "standards." We obtained 94 observations of $\tau$ Ceti with Dewar 13; 869 observations with Dewar 6; and 939 observations with Dewar 8. Consistent with the results for the other standard stars, we found that the median velocity of Dewar 13 observations were greater by $0.88 \mathrm{~m} \mathrm{~s}^{-1}$ than the median velocity obtained with Dewar 6 , 
and the median of the Dewar 8 velocities were $13.6 \mathrm{~m} \mathrm{~s}^{-1}$ lower than the median velocities for Dewar 6.

Since the calculated offset between velocities obtained with Dewar 13 and Dewar 6 is well below our measurement error $\left(-0.37 \mathrm{~m} \mathrm{~s}^{-1}\right.$ for the set of 18 radial velocity standard stars and $+0.88 \mathrm{~m} \mathrm{~s}^{-1}$ for $\tau$ Ceti) we have not made any corrections to the Dewar 13 velocities. However, we adopted a zero-point correction of $+13.1 \mathrm{~m} \mathrm{~s}^{-1}$, added to Dewar 8 velocities for all of the Lick planet search stars. We note that the existence of zero-point offsets and our best effort corrections represent systematic errors that can compromise our ability to detect low-amplitude, long-period exoplanets in the Lick data.

The high resistivity CCD in Dewar 8 introduced some other problems. The 200-micron silicon wafer presented a wider cross-section for interactions with cosmic-ray muons; instead of pinpoint cosmic ray events, longer tracks were observed. In addition, the detector is sensitive to ambient gamma radiation, which produces Compton scattered electrons that random walk in wormlike trails through the detector (Smith et al. 2002). Sources for the gamma radiation include concrete, bedrock, and even the BK7 glass in dewar windows. In an attempt to reduce the gamma ray flux at the detector the dewar was wrapped with lead shielding. Unfortunately, the lead shielding turned out to be a source of gamma radiation and the number of recoiling electron tracks actually increased. Other shielding materials were tested without a significant improvement, so that in the end, we used the dewar without shielding. As a result the low intensity electron tracks had the effect of slightly degrading the SNR. Since the number of Compton scattering events scaled as the exposure time, the quality of spectra was most affected for faint stars or for the typically longer (i.e., 20minute) observations on the CAT. There were also minor problems with chuck patterns on the substrate of the detector that did not quite flat field out, drifts in voltage that changed the width of the SLSF, and the thicker detector showed up to $20 \%$ fringing, increasing for orders redward of the iodine region. The mean single measurement (unbinned) error for $\tau$ Ceti from the Dewar 8 data was $2.65 \mathrm{~m} \mathrm{~s}^{-1}$ and the RMS over the ten year period from 2001 to 2011 was $7.56 \mathrm{~m} \mathrm{~s}^{-1}$. Scatter of $7 \mathrm{~m} \mathrm{~s}^{-1}$ was typical for long time series Doppler measurements of standard stars at Lick and was not considered to be indicative of intrinsic dynamical velocity variations in the $\tau$ Ceti data set.

\section{Radial Velocity measurements for the Lick Sample}

The stellar sample initially consisted of 120 bright FGK and M dwarfs, drawn from

the Bright Star Catalogue (Hoffleit \& Jaschek 1982) and the Gliese-Jahreiss catalog (Gliese 1969; Gliese \& Jahreiss 1979). Binary stars with separations smaller than a few arc seconds 
were excluded from the sample because the flux contamination from a second star would complicate the Doppler modeling. In 1997, many of the fainter stars were moved to the Keck program and roughly 200 new stars were added at Lick. Since most of the stars that were moved to the Keck program had $B-V>0.8$, the Lick planet survey was dominated by brighter and hotter stars. This can be seen in Figure 6 where the $B-V$ distribution is plotted. Most of the stars in the Lick sample had $0.4<B-V<0.7$. These earlier spectral types contributed to the Doppler errors since the late $F$ and early $G$ dwarfs have more intrinsic stellar variability (Isaacson \& Fischer 2010) and tend to be more rapidly rotating.

Motivated by the emerging correlation between stellar metallicity and the formation of gas giant planets, (Gonzalez 1997; Santos et al. 2004; Fischer \& Valenti 2005), an additional 60 metal rich stars were added to the program in 2001, bringing the total sample size to 387 stars (Fischer et al. 1999). As discussed by Fischer \& Valenti (2005), adding metal-rich stars to the sample does not induce a planet-metallicity correlation because the fraction of stars with planets is calculated for each metallicity bin. Rather, the Poisson error bars are reduced for any metallicity bins containing a larger numbers of stars.

Two other planet surveys were also carried out at Lick: a search for planets orbiting nearby K giants (Frink et al. 2001), which grew out of a study for the Space Interferometry Mission, and a Ph.D. thesis survey for planets around subgiants (Johnson et al. 2006); this paper does not include the data from those two projects.

Table 1 lists the stars on the long-term Lick planet search program. In the first column the star identifier is given. The next two columns contain the right ascension and declination for epoch 2000. The fourth column contains the visual magnitude of the stars. The fifth column lists spectral types that are drawn from the Hipparcos catalog (ESA 1997) when available, or listed on SIMBAD. The last three columns list the number of observations, the time interval of the observations in years, the median error for the data set, and the velocity RMS. The print version of the paper contains a stub table listing the first few entries of the complete table that is available online in machine readable format. Figure 7 shows the distribution of both the single measurement errors (tilted cross-hatched part of the histogram) and the RMS velocity scatter (vertical lined histogram) for the full time baseline for each star. In some cases, large RMS scatter occurs because the stars have exoplanets or stellar companions (no trends or Keplerian models were removed when calculating the velocity RMS for Table 1 or Figure 7).

Table 2 contains the individual radial velocities for the stars listed in Table 1 . A stub table is shown in the printed version of the journal, but the online paper contains the complete table in machine readable format with the star name (given as an HD number when available), the observation date in JD - 2440000., the measured relative velocity in 


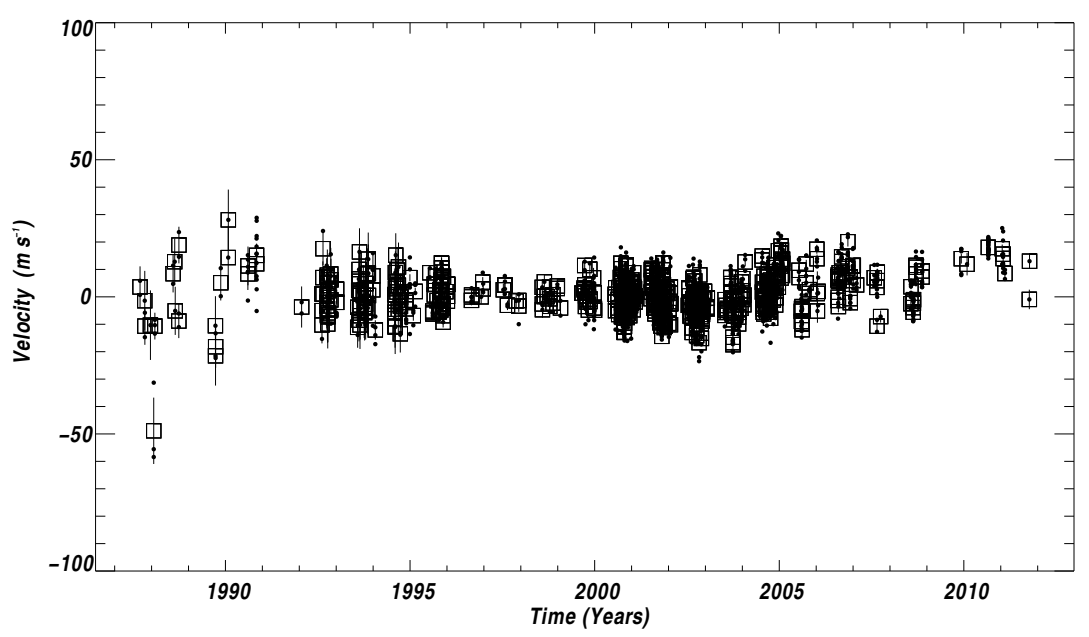

Fig. 1.- Twenty five years of Doppler measurements for $\tau$ Ceti from Lick Observatory. The RMS for this complete data set is $7.9 \mathrm{~m} \mathrm{~s}^{-1}$ and the mean error is $1.7 \mathrm{~m} \mathrm{~s}^{-1}$. This represents an example of low RMS scatter for a star on the Lick Planet Search.

Table 1. Star sample

\begin{tabular}{lcccccrrrr}
\hline \hline $\begin{array}{c}\text { Star ID } \\
\text { ID }\end{array}$ & $\begin{array}{c}\text { RA } \\
(2000)\end{array}$ & $\begin{array}{c}\text { Dec } \\
(2000)\end{array}$ & Vmag & $\begin{array}{c}\text { B-V } \\
\text { Spectral } \\
\text { Type }\end{array}$ & $\begin{array}{c}\text { Nobs } \\
{[\text { Time obs }]}\end{array}$ & $\begin{array}{c}\text { Median Err } \\
{\left[\mathrm{m} \mathrm{s}^{-1}\right]}\end{array}$ & $\begin{array}{c}\text { RV RMS } \\
{\left[\mathrm{m} \mathrm{s}^{-1}\right]}\end{array}$ \\
\hline 166 & 000636.78 & +290117 & 6.13 & 0.750 & K0 & 57 & 24.33 & 7.73 & 21.57 \\
400 & 000840.93 & +363737 & 6.22 & 0.456 & F8 & 19 & 13.19 & 6.86 & 24.67 \\
984 & 001410.25 & -071156 & 7.33 & 0.480 & F5 & 3 & 1.14 & 19.66 & 210.62 \\
gl14 & 001706.37 & +405653 & 8.94 & 1.420 & K7 & 15 & 10.25 & 12.56 & 16.04 \\
gl15a & 001822.88 & +440122 & 8.07 & 1.560 & M1.5 & 25 & 9.88 & 13.73 & 20.21 \\
\hline
\end{tabular}

Note. - This is a stub table with the first few entries of the machine readable table (MRT) that lists parameters for 386 stars. The MRT is available with the electronic version of this paper 


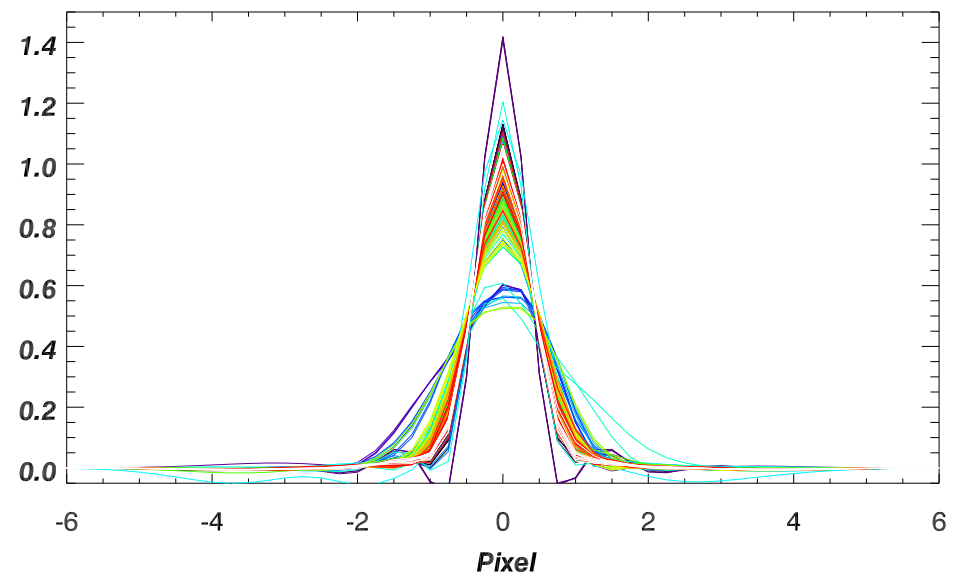

Fig. 2.- SLSF models are over plotted for a single central chunk in the iodine wavelength region. The models were fit to observations of all B stars observed between 1995 and 1997 using the CCD in Dewar 13 on the Hamilton spectrograph. Two families of modeled SLSF result from using the CAT (narrower) and the Shane 3-m (broader). Considerable variation in the SLSF is apparent, typical of slit-fed spectrometers.
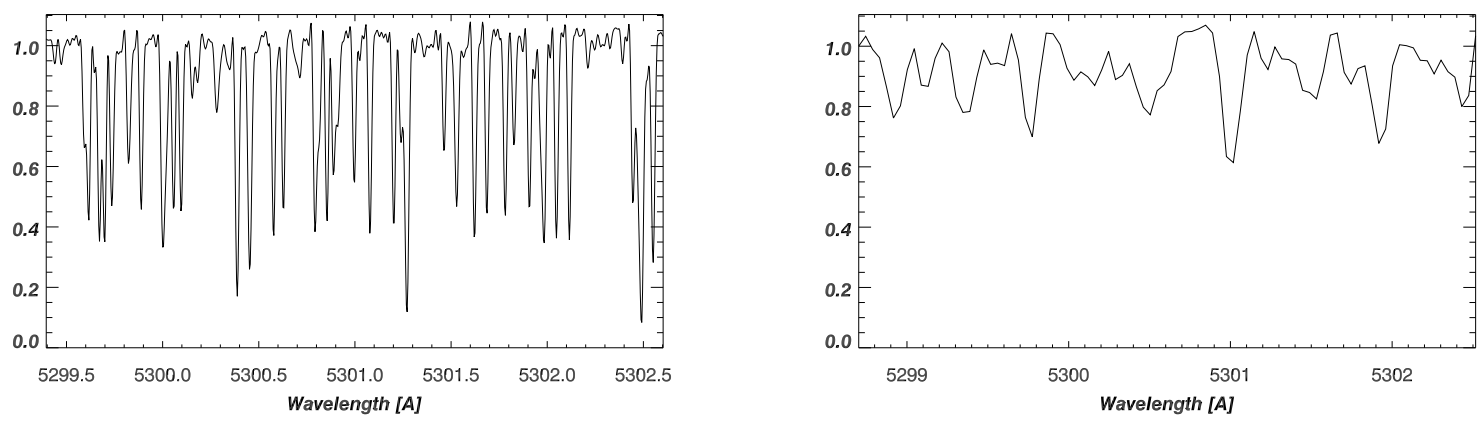

Fig. 3.- (left) the FTS high resolution spectrum of iodine is plotted with flux as a function of vacuum wavelengths and (right) the observed spectrum for that same wavelength segment when observed with the Hamilton spectrograph using the CCD in Dewar 13 (plotted with a wavelength solution derived from Thorium-Argon lamp calibration. The observed spectrum on the right is a convolution of the FTS spectrum with the SLSF shown in Figure 2. 


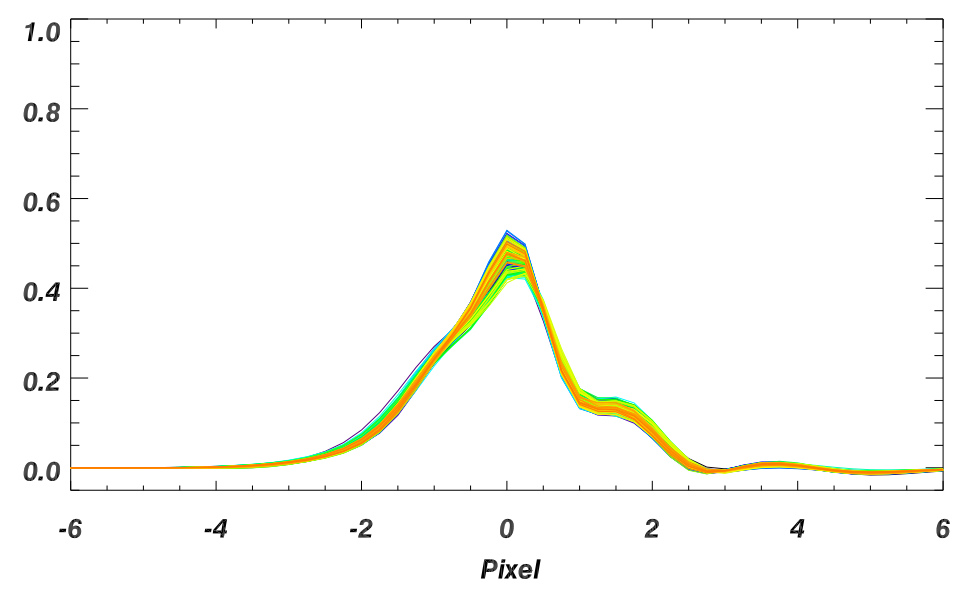

Fig. 4. - SLSF models are overplotted for a single central chunk in the iodine wavelength region. The models were fit to observations of all B stars observed between 1997 and 2001 using the CCD in Dewar 6 on the Hamilton spectrograph.

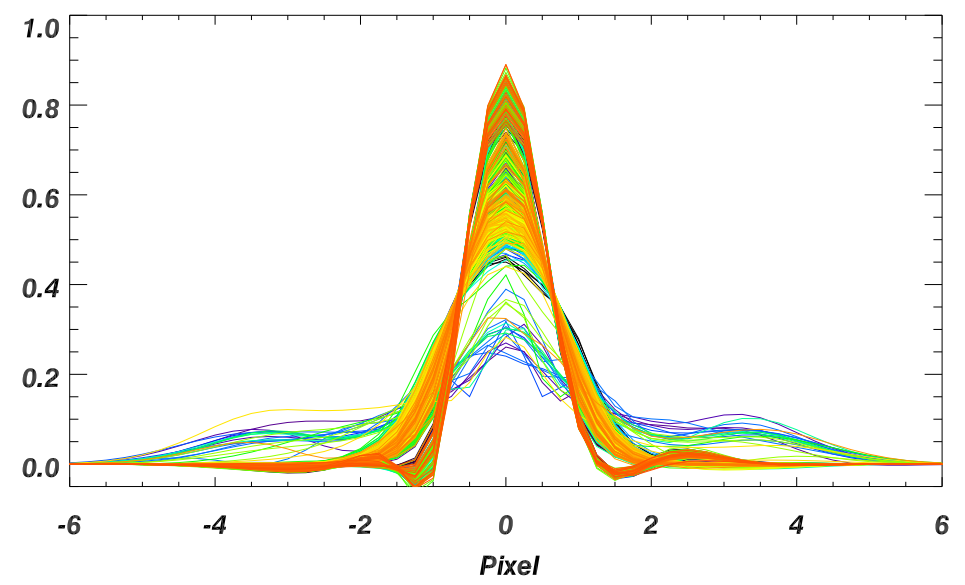

Fig. 5.- SLSF models are over plotted for a single central chunk in the iodine wavelength region. The models were fit to observations of all B stars observed between 2001 and 2011 using the CCD in Dewar 8 on the Hamilton spectrograph. 


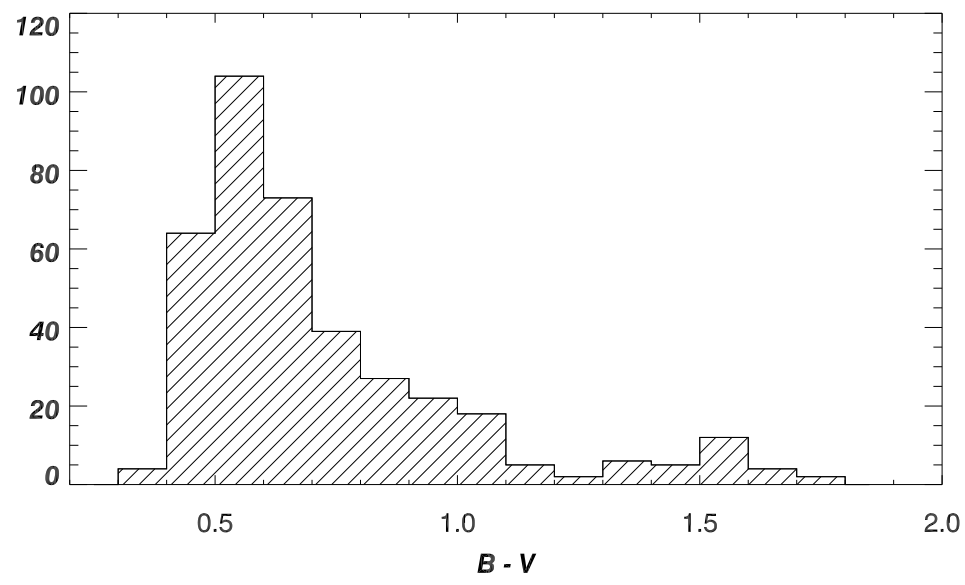

Fig. 6. - The distribution of B-V colors for stars on the Lick planet search sample shows a selection bias toward brighter and relatively bluer stars.

Table 2. Table of Radial Velocity Measurements

\begin{tabular}{ccrrrc}
\hline \hline $\begin{array}{c}\text { Star } \\
\text { ID }\end{array}$ & $\begin{array}{c}\text { JD - 2440000. } \\
\text { [days }]\end{array}$ & $\begin{array}{c}\text { RV } \\
{\left[\mathrm{m} \mathrm{s}^{-1}\right]}\end{array}$ & $\begin{array}{c}\text { Error } \\
{\left[\mathrm{m} \mathrm{s}^{-1}\right]}\end{array}$ & SNR & Dewar \\
\hline 166 & 6959.98020 & 7.95 & 12.65 & 269 & 2 \\
166 & 7046.88100 & 3.05 & 8.95 & 277 & 2 \\
166 & 7373.92230 & 25.78 & 7.73 & 240 & 2 \\
166 & 7846.79660 & 17.41 & 9.13 & 224 & 2 \\
166 & 8113.92840 & 6.84 & 11.68 & 307 & 2 \\
\hline
\end{tabular}

Note. - This is a stub table with the first few entries for one star. The machine readable table available with the electronic version of this paper lists more than 14,000 velocity measurements for 386 stars. 


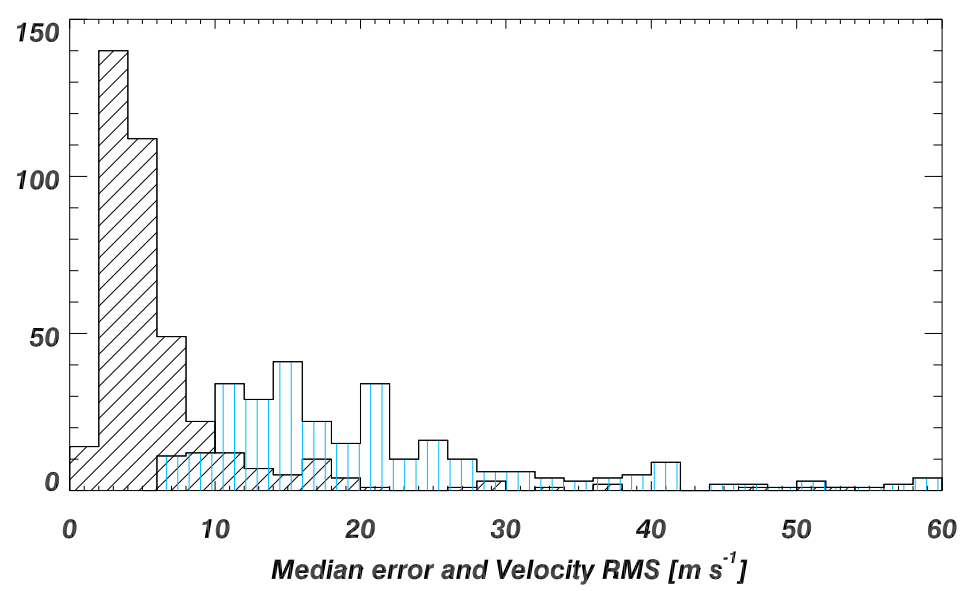

Fig. 7.- The distribution of Doppler errors (angled, cross-hatched histogram) peaks at 2 - 4 $\mathrm{m} \mathrm{s}^{-1}$. However, most stars exhibit RMS radial velocity scatter (vertical-lined histogram) of tens of meters per second. This is because the sample includes some stars with exoplanets, a large fraction of late $\mathrm{F}$ or early $\mathrm{G}$ type stars that are rapidly rotating and have significant stellar jitter (astrophysical noise sources) and because of uncontrolled systematic errors. 
units of $\mathrm{m} \mathrm{s}^{-1}$, the formal measurement error in units of $\mathrm{m} \mathrm{s}^{-1}$, the SNR per pixel near blaze center of a central iodine order, and the CCD dewar that was used for the observation. The zero-point correction discussed in Section 2.6 has been added to the velocities obtained with Dewar 8.

\section{Testbed for Innovation}

\subsection{Tip-Tilt at the Coudé Focus}

The 3-m Shane telescope has a long focal length and is subject to wind shake. On many nights, the image of the star spends less than $50 \%$ of the time on the slit. In Fall 1997, we built a prototype tip-tilt system in front of the slit to the Hamilton Spectrograph (Eiklenborg 1998). The system was mounted on an optical table where a 45-degree fixed position pick-off mirror directed the starlight to a tip-tilt mirror mounted on a stepper motor driven gimbal mount. The light was then directed back toward the slit by a second 45-degree fixed mirror. A beam splitter directed about $4 \%$ of the light to a quadrant photo-multiplier tube (PMT) and a signal from each quadrant was used to close the loop by moving the tip-tilt mirror so that all PMT quadrants had equal amounts of light. The prototype was tested on three nights and demonstrated an improvement in throughput, particularly on one windy night when the open loop observations suffered large light losses at the slit. However, the RMS scatter of the radial velocities for a set of observations was marginally worse than a control case with an open loop. The results were not conclusive because the number of observations was small, however, one concern is that the tip-tilt mirror was changing the angle of light and therefore changing the illumination of the optics in the spectrometer. A better approach would be to use the tip-tilt system with a fiber scrambler.

\subsection{Tests with Fiber Scramblers}

In a series of tests performed with the slit-fed Hamilton spectrograph (Spronck et al. 2013), we have shown a strong correlation between SLSF variations and changes in star position in the sky, most likely due to changes of spectrograph illumination with varying telescope positioning (Figure 8; Spronck et al. 2013). In addition, we have also documented correlations between SLSF variations and guiding, focusing and seeing changes. These tests demonstrated that slit-fed spectrometers are unlikely to provide the extraordinary stability required in order to achieve extreme radial velocity precision.

In an effort to control short timescale variations in the SLSF, Spronck et al. (2013) 


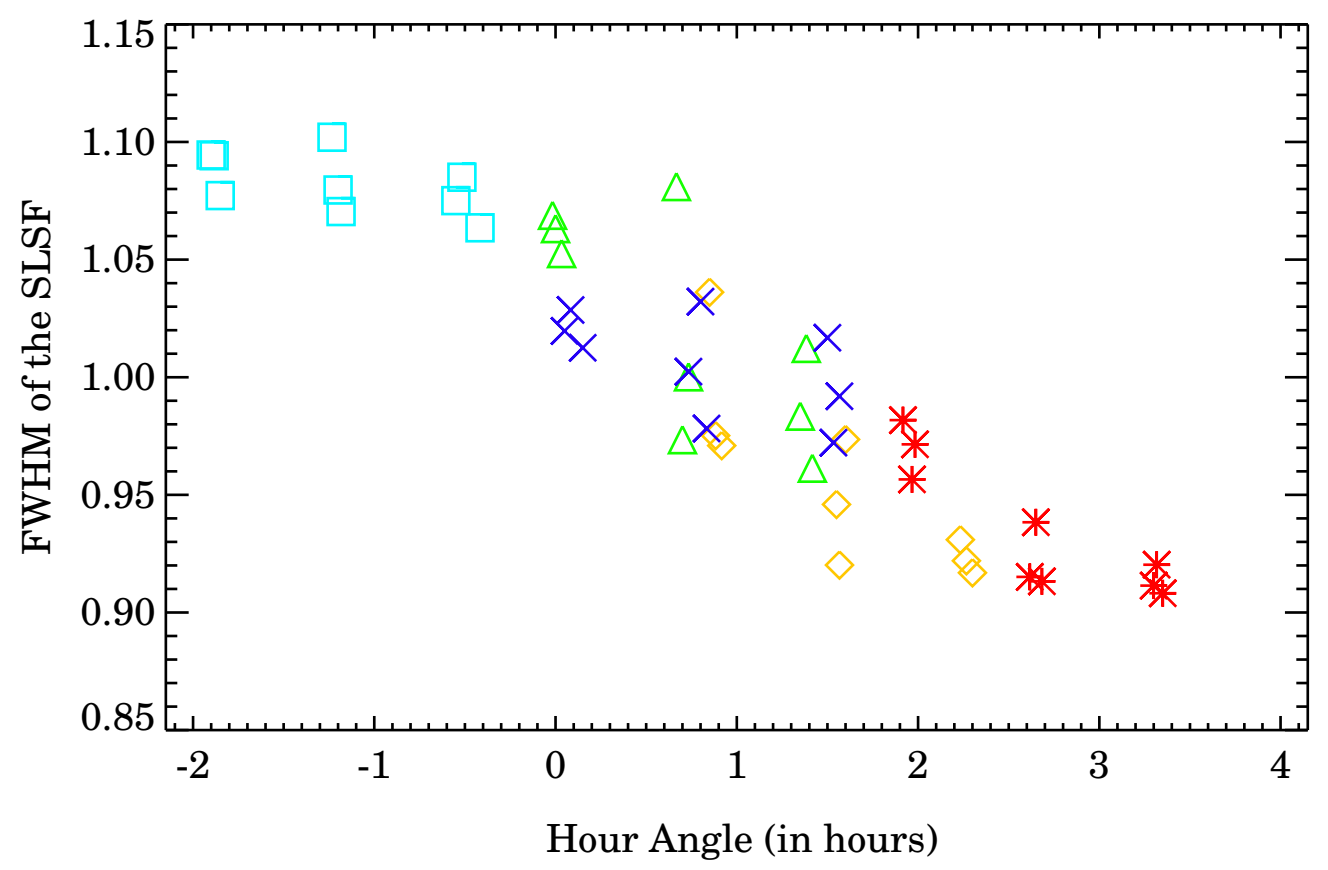

Fig. 8. - SLSF for $45 \mathrm{~B}$ star observations as a function of hour angle (reproduced Figure 8b from Spronck et al. 2013). Each colored symbol corresponds to a different B star. 
designed a prototype fiber scrambler to feed light to the Hamilton Spectrograph. The iodine cell is located about $10 \mathrm{~cm}$ in front of the decker plate with space for a pickoff mirror to feed the TV guide camera. Because of these space considerations and because we wanted to use the existing TV target acquisition and guiding system, the fiber scrambler was mounted on an optical breadboard behind the entrance aperture to the spectrometer. Tests were made with a 100 micron circular fiber that was 15 meters in length and the throughput of the fiber scrambler was measured to be $65 \%$.

Our analysis of spectra from the B star illuminated iodine cell showed that the variability of the SLSF improved by a factor of two when using the fiber (Spronck et al. 2013). However, a systematic trend in the full-width half maximum (FWHM) of the SLSF was observed as a function of hour angle, even when using the fiber scrambler. This suggests incomplete scrambling by the fiber that resulted in output that was dependent on the angle that light entered the fiber.

For test observations of HD 166620 with comparable SNR in both the fiber and the slit observations, we found that the velocity RMS decreased by about $30 \%$ for the fiber observations. For fiber observations of two other test stars, the velocity was a few percent worse. However, the SNR was about $50 \%$ lower for all of the fiber observations relative to the slit observations, making a controlled comparison impossible and biased against good results for the fiber observations.

A double scrambler (Hunter \& Ramsey 1992) was also tested with the Hamilton spectrograph. In the double scrambler, the near field image of the fiber is inverted into the far field (pupil) that is then injected into a second fiber. The output from the second fiber then illuminates the spectrograph. The alignment of this system was not optimized and the throughput dropped to $15 \%$. Yet, the SLSF stability improved by another factor of two and the trend in FWHM of the SLSF with hour angle that we observed with the single round fiber was eliminated with the double scrambler.

Although the fiber scrambler was only used in engineering mode at Lick Observatory, these tests provided valuable information about optimal coupling of light to spectrometers for future designs.

\section{3. $\quad$ FTS iodine scans}

In addition to hardware upgrades, we also worked to improve our data analysis techniques. The Fourier Transform Spectrograph (FTS) scan of the iodine cell is perhaps the single most important component in our Doppler analysis because it affects every stage of 
the modeling process. The FTS iodine spectrum is supposed to provide "ground truth" for determining the wavelength solution, measuring Doppler shifts, and modeling the SLSF. The FTS iodine spectrum is also important for generating a second key ingredient in our model: the intrinsic stellar spectrum (ISS). As discussed in Section 2.1, the ISS is created by deconvolving a stellar template spectrum, a high SNR spectrum without iodine. Since the template spectra do not contain iodine lines, the SLSF used in the deconvolution is derived from observations of the iodine cell taken before and after the stellar template observations.

The original FTS spectrum of the Lick iodine cell was obtained at Kitt Peak and had a resolution of $\mathrm{R} \sim 300000$. We obtained FTS scans at the Environmental Molecular Sciences Laboratory (EMSL) with $\mathrm{R} \sim 1,000,000$ and (per resolution element) $\mathrm{SNR} \sim 1000$. This higher resolution FTS scan should provide a more accurate SLSF model; however, deconvolution techniques tend to amplify any noise and high frequency ringing in the continuum can be seen with some of the deconvolved spectra. An ongoing effort is development of deconvolution techniques with wavelet techniques or low pass filters to attenuate deconvolved shot noise.

\section{Loss of the I2 cell}

On 2011 November 11 we learned that the heater for the iodine cell had malfunctioned, melting most of the foam insulation around the iodine cell. The cell itself remained intact, however, a sticky residue dripped over the optical windows of the cell (Figure 9). The cell windows were carefully cleaned and the insulation was replaced.

When the first spectra were taken with the refurbished cell, we found that the model for the iodine lines yielded an unacceptably poor $\chi^{2}$ fit. We visually inspected the spectrum and found that the iodine lines from spectra obtained after the malfunction did not match those taken before the cell overheated. Furthermore, the differences in the spectra were wavelength dependent.

Figure 10 shows a comparison; an iodine spectrum taken on 2010 April 26 ("ri62.149") before the cell was damaged is plotted in black and an iodine spectrum after the damage, taken in January 2012 ("ri82.49") is plotted in red. To highlight the wavelength dependence of the difference, two plots are shown in Figure 10: the left plot is a wavelength segment at about $5400 \AA$ and the right plot is a wavelength segment at about $5700 \AA$.

Iodine spectra do not normally change and the plotted black spectrum, taken before the cell was damaged, is essentially identical to thousands of iodine spectra of the Lick cell obtained from 1989 through 2011. However, the plotted red spectrum (from the damaged 
cell) in Figure 10 shows significant changes. The spectrum from the damaged cell shows a small wavelength shift at $5400 \AA$ (Fig 10 left) relative to the good iodine spectrum and the spectral lines from the damaged cell are deeper and slightly broader. Progressing to redder wavelengths, the relative shift in the spectral lines from the damaged cell increases as a function of wavelength. Figure 10 (right) shows the comparison for a wavelength segment at about $5700 \AA$ : the spectral lines from the damaged cell lines are significantly shifted and are now shallower than the good iodine spectrum.

This kind of difference in the iodine spectrum was never seen over the many years of the Lick planet search. Before the cell overheated, the same iodine segments looked the same at all wavelengths year after year. To demonstrate that this change was not the result of some other problem in the spectrometer, we obtained a stellar spectrum (without iodine) and compared it to a spectrum of that same star taken a year before. Although there is a difference in the barycentric velocity, the stellar spectrum is unchanged (Figure 11). This test isolated the problem to the iodine cell.

We considered several explanations: iodine or other elements might have been released from the cell wall during heating. Wavelength dependent scattering from the damaged iodine window might have introduced an apparent change to the normalized spectrum. A change in pressure (partial loss of vacuum, but not a loss of iodine) in the cell could have occurred during thermal cycling of the cell. Whatever the cause, the cell was spectroscopically different after the heater malfunction.

We replaced the iodine cell, but realized that this would require a few data points just to calibrate an offset between the old and new velocity measurements. Since we expected that the Automated Planet Finder on a new 2.4-m telescope at Lick would soon come online, we elected to terminate the program on the Hamilton spectrograph.

\section{Discussion}

The Lick planet search project was a pioneering program to measure the reflex velocities induced by exoplanets for a sample of 386 nearby stars. The velocity sets are plotted for all 386 stars on the Lick program in a figure set with the online edition of this paper. Figure 12 shows the first of these figures for the print version of the paper.

Many of the first known planets outside our solar system were identified or confirmed with the Lick survey, including: 51 Peg b (Mayor \& Queloz 1995; Marcy et al. 1997); the first planet in an eccentric orbit, 70 Vir b (Marcy \& Butler 1996); 47 UMa b, c (Butler

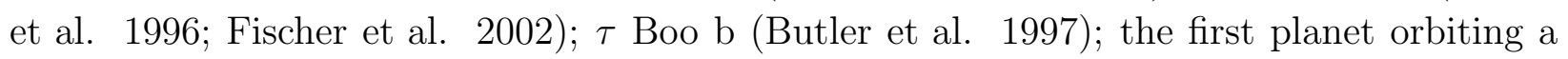


Fig. 9.- Melted insulation dripped across the optical window of the iodine cell after a heater malfunction in 2011 November.
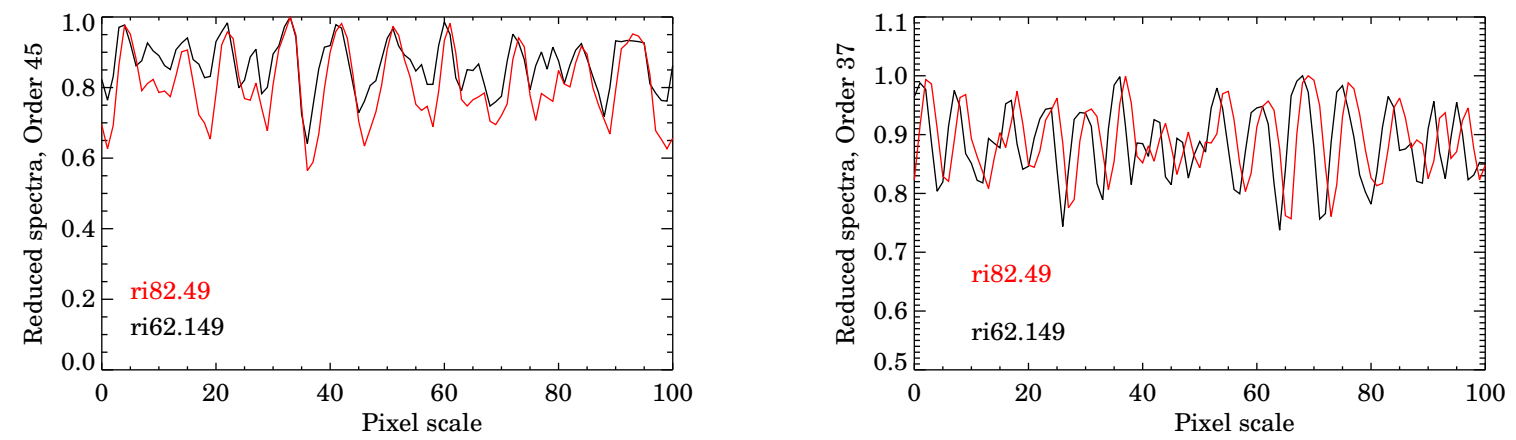

Fig. 10.- A wavelength dependent change was found in the iodine absorption spectrum after the iodine cell overheated. The comparison spectrum is shown in black ("ri62.149") and was obtained before the cell was damaged. This spectrum matches iodine spectra from the previous two decades and is well modeled with the FTS spectrum of the cell. The red spectrum "ri82.49" is an iodine spectrum taken after the heater malfunction and is plotted in red. (left) The damaged-cell spectrum at about $5400 \AA$ is slightly shifted and has deeper and broader lines than the spectrum obtained with the good cell. (right) The wavelength segment at about $5700 \AA$ shows a significant wavelength shift and the spectral line depths are shallower than the (black) good iodine spectrum. 


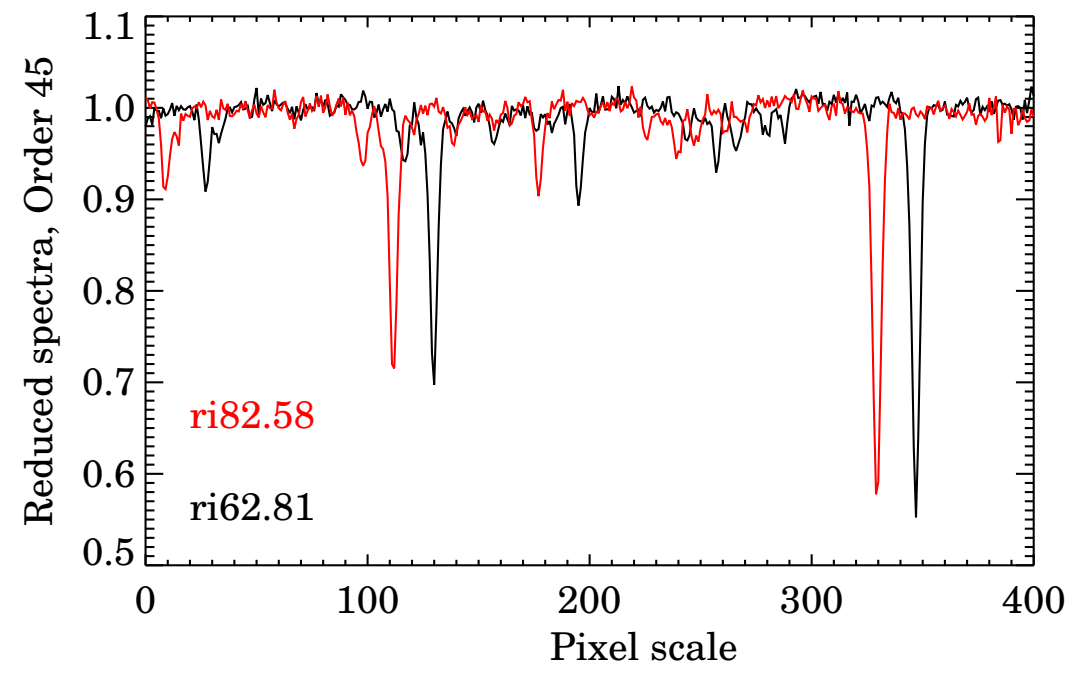

Fig. 11.- A stellar spectrum obtained without the iodine cell on 2010 April 26 ("ri62.81") is unchanged from a spectrum taken after the cell was damaged ("ri82.58") except that the SLSF for the "ri82.58" observation is slightly broader and therefore the lines are a bit shallower. This isolates the observed change in the iodine spectrum to a change in the I2 cell rather than to any changes in the spectrometer or detector. 

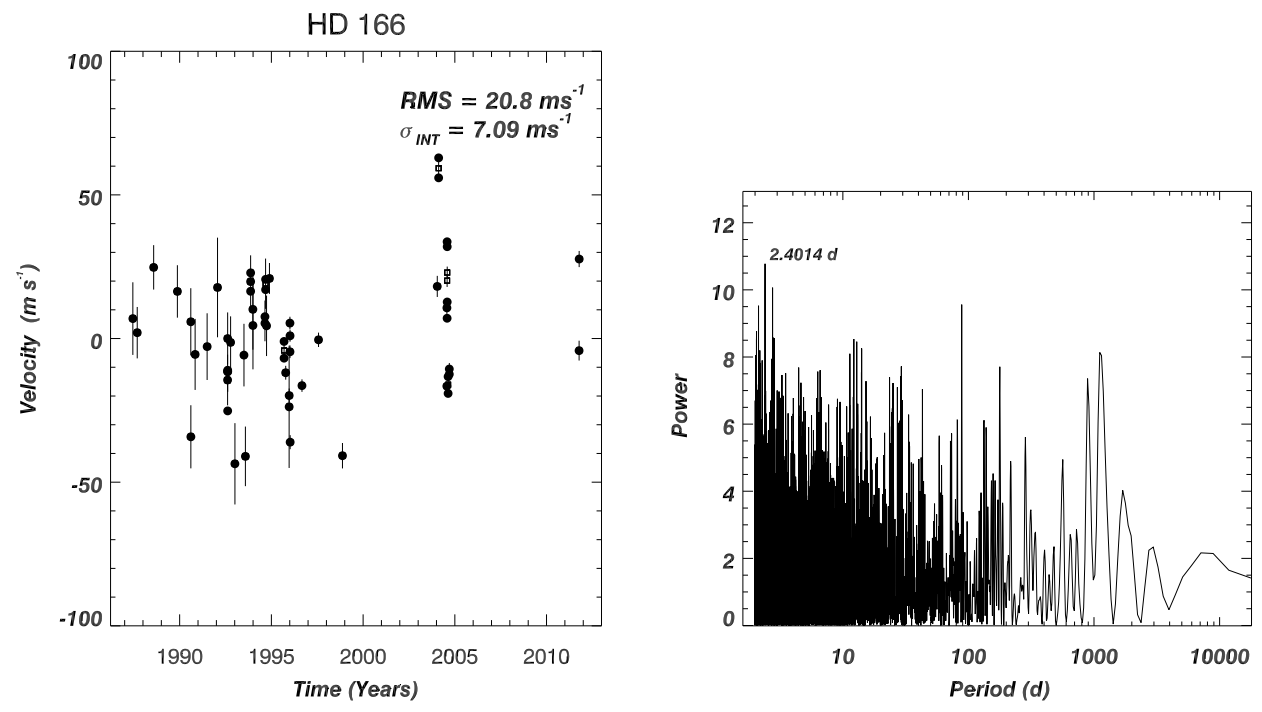

Fig. 12. - Time-series radial velocity data for the star HD 166. This is the first example from the online Figure set plotting Doppler velocities for the 386 stars listed in Table 1. For stars with less than one year of data, the time scale is in Julian days; otherwise the time is in years. The RMS of the velocities as well as the formal measurement error are listed in the top right corner of the plot. Stars with more than 12 observations also have a periodogram plot included as a companion Figure. 
star in a binary system, 16 Cyg B b (Cochran et al. 1996); the first multi-planet system around normal star, Ups And b, c, d (Butler et al. 1997, 1999); 55 Cnc b, c, d, f, g (Butler \begin{tabular}{|l|l|l|l|l|}
\hline et al. 1997; Marcy et al. & $2002 ;$ & Fischer et al. 2008); HD195019 HD217107 (Fischer et al. \\
\hline
\end{tabular} 1999); HD 89744 b (Korzennik et al. 2000); GJ 876 b, c, d (Marcy et al. 2001; Rivera et al. 2005); HD 136118 b, HD 50554 b, HD 105252 b (Fischer et al. 2002); one of the first cold sub-Saturn mass planets, HD 3651 b (Fischer et al. 2003); HD 49079 b, HD 12661 b, c, HD 38529 b, c (Fischer et al. 2001, 2003); HD 30562 b, HD 86264b, HD87883 b, HD 89307 b, HD 148427 b, HD 196885A b (Fischer et al. 2009).

Gas giant planets were found in hot and cold orbits, exoplanets were detected around stars that were members of binary systems, the first multi-planet systems were detected and the project provided data used to quantify the planet-metallicity relation. The Lick program also provided important phase coverage to expedite exoplanet detections at Keck Observatory. At Lick Observatory, we first learned that planet formation is both a robust and chaotic process.

The project served both as a workhorse Doppler survey and as a testbed for innovation. Thanks to the generous allocation of telescope time by the UC TAC, we were able to acquire the first high cadence Doppler data sets and learned that intense sampling was critical for unraveling complex systems with low amplitude and multiple planet signals. We tested a tip-tilt system at the Coudé focus of the telescope and found that while this improved the throughput, particularly on windy nights, the radial velocity precision was slightly degraded - probably because of the changing input angle for light into the Hamilton spectrograph. We also tested fiber scramblers at Lick and achieved significant improvement in the stability of the SLSF. While our velocity precision did not improve significantly with the fiber scrambler, we believe that the error budget was dominated by other factors such as the decreased SNR and slight loss of resolution with the fiber and with instability in the Hamilton spectrograph itself.

We monitored, but were unable to control, large changes in temperature and pressure and significant changes in the SLSF over time for the Hamilton spectrometer. Over the 25year history of the program, we used several different CCD detectors and learned that despite the poor $22 \%$ quantum efficiency of the detector used in 1995 - 1997, the low charge diffusion in that detector resulted in a narrower SLSF and higher Doppler precision. The program was only able to survive all of these changes with what was then state-of-the-art Doppler precision because we used an iodine cell to model the wavelength solution, the Doppler shift, and the SLSF of the instrument. A reference cell (like the iodine cell) is critical for facility instruments that are not stabilized or for instruments where the user community changes the spectrometer configuration. 
During the course of this program, we saw a new state of the art Doppler precision emerge from HARPS (Mayor et al. 2003), a fiber-fed stable instrument. We continued our efforts to improve precision at Lick; one of us (DAF) wrote a new Doppler code and tested new techniques for fitting the data, template deconvolution, and modeling the SLSF. However, even if additional small gains in precision can still be achieved with the spectra obtained from the Lick planet search, the bottom line is that high Doppler precision (better than $1 \mathrm{~m} \mathrm{~s}^{-1}$ ) requires that every detail is right, from the instrument to the analysis code. The Hamilton spectrograph was not designed to deliver sub-meter-per-second precision. To reach much higher precision, the environmental stability (pressure and temperature) need to be controlled, the instrument needs to be stable against vibration and motion, a fiber scrambler is needed to ensure that the illumination of the optics is constant. It is also likely that significant advances in Doppler precision will require a new wavelength calibrator that spans most of the observed spectrum and does not mask "stellar noise," the weak signals imprinted in spectral lines from signals associated with changes in the stellar magnetic field.

We share this legacy of data and lessons learned with the astronomical community and with future planet hunters.

We thank the referee, Artie Hatzes, for helpful comments that have improved this paper. We thank the University of California time allocation committee for supporting this project for more than twenty years. We acknowledge critical pioneering contributions by R. Paul Butler, S. S. Vogt and J. Valenti. We gratefully acknowledge the support and dedication of the Lick Observatory staff, in particular, those who worked side-by-side with us for two decades: Rem Stone, Tony Misch, Keith Baker, Wayne Earthman, John Morey. We thank the many individuals who helped with observing (most were students at San Francisco State University or the University of California, Berkeley); listed roughly in chronological order of their time at Lick, the observers included Eric Williams, Chris McCarthy, Phil Shirts, Mario Savio, Michael Eiklenborg, David Nidever, Amy Reines, Jason T. Wright, Greg Laughlin, Bernie Walp, John Johnson, Eric Nielsen, Melesio Munoz, Howard Isaacson, Julia Kregenow, Matt Giguere, Teresa Johnson, Zoe Buck, Katie Peek, John M. Brewer, Peter Williams, Raj Sareen, Thomas Ader, Kelsey Clubb, Nick van Nispen, Zak Kaplan, Joseph O'Rourke. We also thank Thomas Blake at the Environmental Molecular Sciences Laboratory (EMSL) for help with new FTS scans of the iodine cell. A portion of the research was performed using EMSL, a national scientific user facility sponsored by the Department of Energy's Office of Biological and Environmental Research and located at Pacific Northwest National Laboratory. Fischer acknowledges support by NASA grant NNX10AG08G. Fischer and Spronck thank members of The Planetary Society for support of the fiber scrambler project. This research has made use of the Simbad database, operated at CDS, Strasbourg, France. 
Shane Hamilton spectrograph 


\section{REFERENCES}

Beckers, J. M. 1977, ApJ, 213, 900

Butler, R. P., Marcy, G. W., Williams, E., McCarthy, C., Dosanjh, P., \& Vogt, S. S. 1996, PASP, 108, 500

Butler, R. P., Marcy, G. W., Williams, E., Hauser, H., Shirts, P. 1997, ApJ, 474, 115

Butler, R. P., Marcy, G. W., Fischer, D. A., Brown, T. M., Contos, A. R., Korzennik, S. G., Nisenson, P., Noyes, R. W. 1999, ApJ, 526, 916

Campbell, B.. Walker, G. A. H. \& Yang, S. 1988, ApJ, 331, 902

Campbell, B., Walker, G. A. H. 1985, in IAU Colloquium 88, Stellar Radial Velocities, ed. A. G. D. Philip and D. W. Latham (Schenectady: L. Davis Press), p5

Campbell, B. \& Walker, G. A. H. 1979, PASP, 91, 540

Cochran, W. D., Hatzes, A. P., Butler, R. P., Marcy, G. W. 1996, ApJ, 483, 457

Cochran, W. D. \& Hatzes, A. P. 1990, Proc. SPIE 1318

Dravins, D. 1982 ARA\&A, 20, 61

Dravins, D., Lindegren, L., Nordlund, A. 1981, å, 96, 345

Dravins, D. 1985, in IAU Colloquium 88, Stellar Radial Velocities, ed. A. G. D. Philip and D. W. Latham (Schenectady: L. Davis Press), p311

ESA 1997, The Hipparcos and Tycho Catalogs. ESA-SP 1200

Eiklenborg, M. 1997, MS thesis SFSU http://www.eiklenborg.com/thesis/thesis.htm

Fischer, D. A., Marcy, G. W., Butler, R. P., Vogt, S. S., Apps, K. 1999, PASP, 111, 50

Fischer, D. A., Marcy, G. W., Butler, R. P., Vogt, S. S., Frink, S., Apps, K. 2001, ApJ, 551, 1107

Fischer, D. A., Marcy, G. W., Butler, R. P., Laughlin, G., Vogt, S. S. 2002, ApJ, 564,1028

Fischer, D. A., Marcy, G. W., Butler, R. P., Vogt, S. S., Walp, B., Apps, K. 2002, PASP, 114,529

Fischer, D. A., Butler, R. P., Marcy, G. W., Vogt, S. S., Henry, G. W. 2003, ApJ, 590, 1081 
Fischer, D. A., Marcy, G. W., Butler, R. P., Vogt, S. S., Henry, G. W., Pourbaix, D., Walp, B., Misch, A., Wright, J. T. 2003, ApJ, 586, 1394

Fischer, D. A. \& Valenti, J. 2005, ApJ, 622, 1102

Fischer, D. A., Marcy, G. W., Butler, R. P., Vogt, S. S. Laughlin, G., Henry, G. W., Abouav, D., Peek, K. M. G., Wright, J. T., Johnson, J. A., McCarthy, C., Isaacson, H. 2008, ApJ, 675, 790

Fischer, D. A., Driscoll, P., Isaacson, H., Giguere, M., Marcy, G. W., Valenti, J., Wright, J. T., Henry, G. W., Johnson, J. A., Howard, A., Peek, K., McCarthy, C. 2009, ApJ, 703,1545

Frink, S., Quirrenbach, A., Fischer, D., Röser, S., Schilbach, E. 2001, PASP, 113, 173

Gliese, W. 1969, Veröff. Astron. Rechen-Inst. Heidelberg, No. 22

Gliese, w., Jahreiss, H. 1979, A\&AS, 38, 423

Gonzalez, G. 1997, MNRAS, 285, 403

Gray, D. F. \& Toner, C. G. 1985, PASP, 97, 543

Griffin, R. and Griffin, R., 1973, MNRAS, 162, 243

Hatzes, A. P., Cochran, W. D., Endl, M., McArthur, B., Paulson, D. B., Walker, G. A. H., Campbell, B., Yang, S. 2003, ApJ, 599, 1383

Hoffleit, D. \& Jaschek C. 1982, The Bright Star Catalogue (4th rev. ed; New Haven: Yale Univ. Obs.)

Hunter, T. R. \& Ramsey, L. W. 1992, PASP, 104, 1244

Isaacson, H., Fischer, D. A. 2010, ApJ, 725, 875

Johnson, J. A., Marcy, G. W., Fischer, D. A., Henry, G. W., Wright, J. T., Isaacson, H., McCarthy, C. 2006, ApJ, 652, 1724

Kibrick, R. I., Clarke, D. A., Deich, W. T. S., Tucker, D. 2006, Proc. SPIE 6274, 58

Korzennik, S. G., Brown, T. M., Fischer, D. A., Nisenson, P., Noyes, R. W. 2000, ApJ, 533, 147 
Libbrecht, K. G., 1988, in The Impact of Very High S/N Spectroscopy on Stellar Physics, 132nd Symposium of the IAU in Paris, France, June 29 - July 3, 1987, Ed: G. Cayrel de Strobel and Monique Spite. Kluwer Academic Publishers, Dordrecht, p. 83

Libbrecht, K. G., 1989, ApJ, 336, 1092

Mayor, M., Queloz, D. 1995, Nature, 378, 355

Mayor, M. et al. 2003, The Messenger 114, 20

Marcy, G. W., Benitz, K. J. 1989, ApJ, 344, 441

Marcy, G. W., Butler, R. P. 1992, PASP, 104, 270

Marcy, G. W., Butler, R. P. 1996, ApJ, 464, 147

Marcy, G. W., Butler, R. P., Williams, E., Bildsten, L., Graham, J. R, Ghez, A. M., Jernigan, J. G. 1997, ApJ, 481, 926

Marcy, G. W., Butler, R. P., Fischer, D. A., Vogt, S. S., Lissauer, J. J., Rivera, E. J. 2001, ApJ, 556, 296

Marcy, G. W., Butler, R. P., Fischer, D. A., Laughlin, G., Vogt, S. S., Henry, G. W., Pourbaix, D. 2002, ApJ, 581, 1375

McMillan, R. S., Smith, P. H., Frecker, J. E., Merline, W. J., Perry, M. L. 1986, Proc. SPIE V627 in Instrumentation in Astronomy VI.

Noyes, R. W., Baliunas, S. L., Belserene, E., Duncan, D. K., Horne, J., Widrow, L. 1984, ApJ, 285, L23

Rivera, E. J., Lissauer, J. J., Butler, R. P., Marcy, G. W., Vogt, S. S., Fischer, D. A., Brown,T. M., Laughlin, G., Henry, G. W. 2005, ApJ, 634, 625

Santos, N. C., Israelian, G., Mayor, M. 2000, å, 415,1153

Smith, A. R., McDonald, R. J., Hurley, D. L., Holland, S. E., Groom, D. E., Brown, W., Gilmore, D. K., Stover, R. J., Wei, M. 2002, Proc. SPIE 4669, 172.

Spronck, J. F. P., Fischer, D. A., Kaplan, Z. A., Schwab, C., Szymkowiak, A. 2013, PASP, 125,511

Struve, O. 1952, The Observatory 72, 199

Valenti, J. A., Butler, R. P., Marcy, G. W. 1995, PASP, 107, 966 
Vogt, S. S. 1987, PASP, 99, 1214

Walker, G. A. H., Bohlender, D. A., Walker, A. R., Irwin, A. W., Yang, S. L. S., Larson, A. 1992, ApJ, 396, 91 